\title{
PENICILLIN IN SKIN DISEASES
}

\author{
By I. R. MARRE, M.R.C.S., L.R.C.P. \\ Skin Physician to the Metropolitan, Evelina and Acton Hospitals.
}

It is now well recognized that penicillin is no panacea, but nevertheless it is a remarkable drug, and clinicians in every branch of medicine have good reason to be thankful for its usefulness in their own spheres. It is superior to the sulphonamides in that it has a greater range of effectiveness against bacterial diseases, in that it is virtually non-toxic, in that it rarely causes sensitization, in that its action is not inhibited by serum, pus or blood.

Certain bacteria are highly susceptible to penicillin, and others less so, while there is unfortunately a long list of insusceptible organisms.

The Medical Research Council ${ }^{1}$ list the principal bacteria as follows.

\section{Susceptible}

Gram positive cocci :

Staphylococcus Aureus.

Streptococcus Pyogenes.

Other streptococci (except enterococcus).

Pneumococcus.

Clostridia :

Cl. welchii.

Cl. septicum.

Cl. oedematiens.

Cl. tetani.

Neisseria :

$\mathrm{N}$ : gonorrhoeiae.

N. meningitidis.

Miscellaneous :

B. anthracis.

Actinomyces bovis (variable).

C. diphtheriae.

\section{Insusceptible}

Proteus.

Ps. Pyocyanea.

Coli-typhoid-dysentery group.

Pasteurella and Brucella.

Str. faecalis (enterococcus).

M. tuberculosis.
To these should be added Treponema Pallidum and Spirochaetes (susceptible) and Monilia, Yeasts and Moulds (insusceptible).

It will thus be seen that most of the gram negative bacteria are insusceptible, except for the gonococcus and the meningococcus, but that the main pyogenic cocci, which are the usual cause of septic skin diseases, are highly susceptible. The viruses are unaffected by penicillin, and there seems no point in using the drug in conditions such as herpes zoster and herpes simplex. Indeed I shall show later that in the latter disease it may, actually be unwise to use penicillin. Again in allergic and constitutional skin conditions, unless infected, one would expect no favourable response and this is in fact what happens. The fungus infections form a large group of skin troubles which are also unaffected by penicillin.

Penicillin may be administered in a number of ways. Injections may be given intravenously, intramuscularly or subcutaneously, either by continuous drip or three-hourly intermittent injections. The three-hourly injections are given in aqueous solution of 15,000 to 20,000 units. For most skin diseases it is sufficient to administer penicillin, where injection is indicated, twice daily, either 300,000 units in aqueous solution per dose, or 150,000 units in oil and wax suspension. My own preference is to give aqueous solutions as there seems to be a rather more rapid clinical response and no liability to local reactions from the suspension which occurs occasionally. Local infiltration and injection have been described, especially for abscesses and carbuncles, but I prefer general administration.

Local treatments are of particular importance in skin diseases and penicillin may be applied by means of creams, ointments and sprays. Creams and sprays are fairly unstable and have to be kept in a cool place or in a refrigerator, and renewed at least every seven days. Cremor penicillini B.P., is a lanette wax 
cream made up to 500 units per gramme, but I prescribe the cream made up to $I, 000$ units per gramme, and I think the results are better. The spray should be made up in sterile water at the same strength ( $\mathrm{I}, 000$ units per c.c.), and kept in an all glass spray, which should be boiled between changes of penicillin solution. Penicillin ointment B.P., is an ointment of wool alcohols (500 units per gramme) which is said to keep for months at room temperature, owing to its anhydrous constitution. This is a greasy ointment and must be used with care, and again $I$ use this at double strength.

Although the spray is perhaps the most difficult to keep at home, I find it the most effective treatment if used often enoughthree or four times daily, and allowed to dry on. It is certainly very effective in the superficial coccal infections. All the preparations should not be contaminated with the finger or an unsterile spoon or spatula, for fear of introducing insusceptible organisms. Cream or ointment can be removed from the container with some instrument which has been held in boiling water, and then cooled rapidly in the air. If crusting is severe, this may be gently removed by oil packs or boro-starch poultices, ${ }^{2}$ but mild crusting may be left to dry or fall off in the course of treatment.

Penicillin appears to be absorbed by the gastro-intestinal tract, but to obtain any sort of concentration in the blood it is necessary to give very large quantities, at least five times that by injection. The method is uncertain, and is not used routinely, although work is being carried out in this direction. Mouth infections do respond to lozenges (Trochiscus Penicillini B.P.), which contain 500 units per gramme, but persistence with this form of treatment for more than a few days results in a glossitis.

Cultures of organisms from septic skin areas should be routine in hospital, together with testing for penicillin sensitivity, but treatment of severe and acute cases should not be held up on this account, but should be started as soon as the culture has been taken. In general practice it will be difficult to do this, and, in view of the fact that most pyodermias are caused by sensitive organisms, full treatment may be given at once.

\section{Impetigo Contagiosa}

This extremely common disease is to be found in infants as impetigo (pemphigus) neonatorum, a bullous staphylococcal eruption which spreads rapidly, and is very contagious. In older children and adults the typical streptococcal impetigo with its honey-coloured 'stuick-on' crusts is more usual, or the deeper bullous staphylococcal type. Early cases respond well to local therapy in the shape of cream or ointment applied three times daily, but in more severe cases injections as well may be needed. In impetigo neonatorum careful snipping of the blisters before applying the cream aids recovery. Improvement should be noted after the first day or two, and recovery should be almost complete within five or six days. If penicillin has had no effect in this time, the treatment should be changed, as the organism is likely to be insensitive. It should be borne in mind that impetigo is often secondary to scabies, pediculosis capitis or vestimentorum, a discharging ear or some other focus of infection, and that the impetigo will recur unless the primary condition is treated subsequently. All cases of impetigo should be carefully investigated for infestation or foci of infection.

\section{Furunculosis}

Boils are staphylococcal abscesses originating in and around a hair follicle. Carbuncles may be described as a group of boils in close proximity, becoming one large circumscribed mass with a few openings through which pus discharges. Where the boil is single and small, local application of penicillin cream or ointment may effect a cure, and will prevent the spread of secondary boils in the area. Where boils are multiple and large, systemic penicillin should be administered, possibly accompanied by local treatment as well. After the attack has been dealt with, an autogenous or stock staphylococcal vaccine is of great value in raising resistance against the organism, and should be given routinely in a case of any severity. The patient should also be thoroughly examined for any systemic disorder rendering him more liable to septic infection, such as diabetes, nephritis, dysentery or general debility. Septic foci should be sought and dealt with if present, and tonics prescribed. Pedicu- 
losis capitis is a common cause of boils on the scalp, neck and back in school girls and even in women.

\section{Sycosis Barbae}

This is a staphylococcal folliculitis of the bearded area, and is often known as barber's rash. The response to this disease is on the whole good, and local treatment is usually sufficient, unless the degree of infection is unusually severe. For the purposes of treatment it is sufficient to divide sycosis into three groups.

(a) Primary infection, or following on a superficial infection such as impetigo.

(b) Infection secondary to other local foci, particularly septic conditions of the mouth and gums, of the nose and nasal sinuses, and of the ear.

(c) Infection following on seborrhoeic dermatitis.

Local treatment with the first group usually shows a dramatic response with little tendency to recur unless the condition is long standing, and the deeper parts of the hair follicle have become infected. In these more chronic conditions treatment must be persisted with for weeks or even months, and for some time after apparent cure, and the final result is satisfactory. In the second group an equally satisfactory result is obtained temporarily, but unless the primary focus is appropriately treated the condition will of course recur. In the third group the secondary folliculitis shows great improvement for a few days, but frequently at this stage the seborrhoeic condition (see seborrhoeic dermatitis below) becomes exacerbated by the local application of penicillin with the production of an acute dermatitis. In cases therefore with a primary seborrhoeic basis, after four or five days of local treatment with penicillin, vigorous antiseborrhoeic treatment ${ }^{3}$ will complete the cure.

\section{Acne Vulgaris}

Acne is a constitutional disease, and does not itself respond to penicillin. In the infected, pustular types of acne a short preliminary course of local treatment is useful to clear up secondary infection, but must be immediately followed up by attention to the acne.

\section{Seborrhoeic Dermatitis}

This too is a constitutional disease, and one would not therefore expect it to respond to penicillin. In many cases there is no effect, but in a number of cases, patients with this condition show a marked sensitivity to penicillin, with an acute exacerbation of the disease. Where the seborrhoeic dermatitis is infected it is justifiable to use local penicillin for a few days only, to be followed up by the usual seborrhoeic treatments. It is my practice never to use penicillin in uncomplicated seborrhoeic dermatitis, and; when infected, only under the strictest supervision and for a limited period.

\section{Otitis Externa}

This common and acutely uncomfortable condition has not, in my hands, responded to penicillin, either by local or general medication, or both. Many of the cases are associated with a seborrhoeic dermatitis and on that account do not respond. In others there is an associated otitis media which needs attention. The largest group, however, has neither middle ear disease nor seborrhoeic dermatitis, and is often complicated by recurrent furuncles in the meatus, but nevertheless does not seem to respond to penicillin, possibly because of the great meatal swelling and interference with the blood supply. I find packing and the antiseptic dyes a more satisfactory treatment.

\section{Echthyma}

This is not an uncommon condition found usually in children suffering from malnutrition, but occasionally in adults as well. It is seen as isolated lesions chiefly on the legs and thighs, commencing as a reddened papule which becomes vesicular and then covered with a dirty looking crust. When this falls off or is removed, a small superficial indolent ulcer is formed, often surrounded by an inflammatory halo, and healing is usually slow. If the crusts are removed, however, and penicillin is applied locally two or three times daily, healing is greatly accelerated with satisfactory results.

\section{Leg Ulcers}

In this country these are mostly due to circulatory disturbances, and particularly to 
varicose veins. I have found penicillin cream of great use in these cases if used for a period of some seven to ten days, when the ulcer becomes much cleaner. After this time I discontinue penicillin and revert to the more usual treatments (always including some form of bandaging to reduce oedema) with good results. To continue for longer than two weeks with penicillin leads, in my experience, to a slowing down of the healing processes. Granulation tissue seems to become inhibited and there is also a tendency for penicillin resistant organisms to grow in the ulcer. Where there is much dermatitis surrounding the ulcer penicillin must be used with care, as occasionally an exacerbation of the dermatitis is caused by the penicillin. Reports ${ }^{4}$ on the treatment of tropical ulcer with penicillin have been very favourable, and this seems to be the treatment of choice in this condition.

\section{Infectious Eczematoid Dermatitis}

This is a condition of acute dermatitis in the form of a plaque or plaques, made up of erythema, vesicles, pustules and crusting and spreading peripherally. The edge is often undermined or raised, and exudes seropurulent fluid. The patient may be undernourished or give a history of some small trauma. The condition often proves resistant to treatment, and although penicillin has improved the position, most of the cases still respond very slowly, and if the condition is at all extensive or affects the legs, treatment is best carried out in bed. I have a personal preference for the antiseptic dyes, together with rest and tonics.

\section{Eczema}

Eczematous eruptions generally of whatever types do not do well on penicillin. Unless infected, conditions such as infantile eczema, exogenous (contact) dermatitis, nummular eczema and the eczematides are either unaffected or exacerbated. Cases have been described $^{5}$ of vesicular eruptions actually occurring during the administration of penicillin, and it is wiser, in the absence of specific indication, to avoid the drug in these cases.

\section{Fungus Diseases}

These appear to be totally unaffected by penicillin, unless secondarily infected. Treat- ment with penicillin of secondary sensitization eruptions due to fungus often leads to an exacerbation of the condition.

\section{Virus Conditions}

Whereas there is some proof that infected virus conditions of the skin may be improved by penicillin, and a case of infected herpes zoster $^{6}$ was so treated, uncomplicated cases of zoster and varicella are unaffected. I have now come across four cases of herpes simplex treated previously with local penicillin where the condition has spread under the influence of the treatment. One case I saw after three weeks of treatment had an extensive herpes simplex spreading over almost half the forehead. In endeavouring to account for this it seemed to me logical to assume not that the virus lived better in an atmosphere of penicillin, but that the normal mild secondary infection which usually occurs when the vesicles rupture, was prevented by the action of the penicillin. It is very possible that the virus is killed by the secondary pyogenic organisms in the normal event, and when this is prevented by penicillin, the virus spreads.

\section{Other Conditions}

Conditions such as psoriasis, lichen planus, Pityriasis rosea and pemphigus, where the aetiology is unknown, are unaffected by penicillin.

\section{Venereal Diseases}

Details of these treatments must be sought elsewhere, but gonorrhoea usually responds dramatically to penicillin, as does the early (treponema positive, sero-negative) case of syphilis. Late primary, secondary and tertiary syphilitics are also given a full preliminary dose of penicillin, with considerable clinical improvement, but this is now usually followed up by treatments of varying lengths with arsenic and bismuth.

\section{Reactions}

These are uncommon but do occur. Some of the reactions have been put down to impurities in the drug, and as the purer penicillin comes to be used, these may become less. True contact dermatitis has been reported, 
' especially with workers with penicillin, but the usual manifestations are erythema, urticaria and a serum sickness type of reaction. Herxheimer reactions have been reported in cases treated for syphilis. Patients with seborrhoeic dermatitis often respond badly to penicillin, as do others with allergic or sensitization eruptions, resulting in an exacerbation of the condition. On a number of occasions, I have seen a local urticaria with penicillin in oil and wax suspensions, but the usual reaction to the drug is a widespread and generalized urticaria, which fortunately responds well to 'Benadryl' capsules (50 mgms. three times daily).

\section{REFERENCES}

I. M.R.C. War Memorandum No. 12. 1944. Reprinted, 1946.

2. MARRE, I. R., 'General Treatment of Skin Diseases,' Post Grad. Med. ̈., Vol. xxiii, No. 255.

3. MARRE, I. R.,' Seborrhoeic Dermatitis,' Post Grad. Med. F., Vol. xxiii, No. 257.

4. HAMM, W. G., and QUARY, G. (1944), 'Penicillin Therapy in Phagedenic Ulcer,' U.S. Nav. M. Bull., 43, 981, November.

5. GRAVES, W. N., CARPENTER, C. C., and UNANGST, R. W. (r 944), 'Recurrent Vesicular E'ruptions Appearing During the Administration of Penicillin,'Arch. Derm. Syph., Vol. 5o, No. I. July.

6. COHEN, T. M., and PFAFF, R. O. (1945), 'Penicillin in Dermatological Therapy,' Arch. Derm. Syph., Vol. 51, No. 3. March.

\section{BOOKS RECEIVED}

The Editorial Board acknowledge with thanks the receipt of the following volumes. $A$ selection from these will be made for review.

' The Rotunda Hospital, I745-1 945.' By O'Donel T. D. Browne, F.R.C.P., F.R.C.O.G. (Pp. 296, 44 illustrations, 42s.) F. \& S. Livingstone Ltd. 1947.

'Mongolism and Cretinism.' By Clemens E. Benda, M.D. (Pp. 330, Io I illustrations, 25s.) Wm. Heinemann. Medical Books Ltd. I 947.

' A Handbook of Diseases of Children.' By Bruce Williamson, M.D., F.R.C.P. 5th F.dition. (Pp. 408, 86 illustrations, ${ }_{5}$ s.) E. \& S. Livingstone Ltd. r 947 .

' Nutritional Disorders of the Nervous System.' By John D. Spillane, M.D., F.R.C.P. (Pp. 280, 2os.) E. \& S. Livingstone Ltd. 1947.

' Medical Diseases in Tropical and Sub-Tropical Areas.' The War Office. 8th Edition. (7s. 6d.) London: His Majesty's Stationery Office. I947.

' History of the Library.' London School of Hygiene and Tropical Medicine, incorporating The Ross Institute. (Pp. 24) Keppel Street, Gower Street, London W.C.I. 1947.

' Clinical Methods of Neuro-Ophthalmologic Examination.' By Alfred Kestenbaum, M.D. (Pp. 384, 65 illustrations, 25s.) Heinemann. Medical Books Ltd. 1947.

'Atlas of Bacteriology.' By R. Cranston Low, M.D., and T. C. Dodds. (Pp. 168, 168 illustrations, 32s. 6d.) E. \& S. Livingstone Ltd. 1947.
'Aids to Qualitative Inorganic Analysis.' By R. G. Austin, B.Sc. 2nd Edition. (Pp. 207, 5s.) Baillière, Tindall and Cox. 1947.

' The Microscope.' By Theodore Stephanides, M.D. (Pp. I60, Ios. 6d.) Faber and Faber Ltd.

Monographs on the Progress of Research in Holland :

'A Contribution to the Knowledge of the Influence of Gonadotropic and Sex Hormones on the Gonads of Rats.' By J. H. Gaarenstroom and S. E. de Jongh. (Pp. I64, I6s.) Published by Elsevier. Distributors Cleaver-Hume Press. 1947.

' Experimental Embryology.' By M. W. Woerdeman, M.D., and Chr. P. Raven, Ph.D. (Pp. I32, 13s.) Published by Elsevier. Distributors CleaverHume Press. 1947.

'Medicine.' Volume I. By A. E. ClarkKennedy, M.D., F.R.C.P. (Pp. 383 , 20s.) E. \& S. Livingstone Ltd. 1947.

'Handbook of Diagnosis and Treatment of Venereal Diseases.' By A. E. W. McLachlan, M.B., Ch.B., D.P.H. 3rd Edition. (Pp. 375, 160 illustrations, I5s.) E. \& S. Livingstone Ltd. 1947.

'Introduction to Dermatology.' By G. H. Percival, M.D., F.R.C.P.E. (Pp. 349, 233 illustrations, 35s.) E. \& S. Livingstone Ltd. I 947.

' Vital Statistics and Public Health Work in the Tropics, including Supplement on the Genealogy of Vital Statistics.' By P. Granville Edge, O.B.E., D.Sc. (Pp. 265) Baillière, Tindall and Cox. I947.

'A Way of Life for the Handicapped Child.' By Eirene Collis.' (Pp. I 83 , Ios. 6d.) Faber and Faber Ltd. I 947. 